\title{
Neumomediastino como causa de llanto
}

\author{
P. Barona Zamora a , J. Marín Serra ${ }^{b}$, S. Pons Morales ${ }^{b}$, R. Margalef Canuto \\ aPediatra. CS Padre Jofré. Departamento de Salud IO. Valencia. España. \\ ${ }^{b}$ Pediatra. 'MIR-Pediatría. Hospital Universitario Doctor Peset. Valencia. España.
}

Rev Pediatr Aten Primaria. 2009; 1 : 283-8

Pascual Barona Zamora, pascualbarona@gmail.com

\section{Resumen}

El neumomediastino es una entidad rara en niños, y su causa principal son las exacerbaciones del asma. Los síntomas más frecuentes son el dolor torácico, que en lactantes y niños pequeños se manifestará como llanto, y la disnea.

Describimos el caso de una niña de 12 meses que presentaba llanto y dificultad respiratoria y que fue diagnosticada de neumomediastino asociado a asma. Destacamos la necesidad de pensar en él ante un cuadro de broncoespasmo con llanto asociado.

Palabras clave: Neumomediastino, Enfisema mediastínico, Asma, Llanto.

\section{Abstract}

The pneumomediastinum is a rare entity in children and its main cause is asthma exacerbations. The most common symptom is thoracic pain that in infants and young children appears as crying, and dyspnoea. We describe the case of a 12-month-old girl who presented with crying and respiratory distress, and was diagnosed of pneumomediastinum associated with asthma. We emphasize the need of suspecting it when we face a case of bronchial obstruction associated with crying.

Key words: Pneumomediastinum, Mediastinal emphysema, Asthma, Crying.

\section{Introducción}

El llanto es un motivo frecuente de consulta en Pediatría. Las causas pueden ser múltiples, desde las fisiológicas o no patológicas hasta las patológicas. Dentro de estas últimas es muy raro que en lactantes o niños pequeños sea por dolor torácico y más aún por un neumomediastino'. La anamnesis y la exploración física nos pueden orientar hacia esta rara entidad y llevarnos a realizar una radiografía de tórax con la que confirmaremos el diagnóstico. Presentamos el caso de una paciente de 12

Los autores declaran no presentar conflictos de intereses en relación con la preparación y publicación de este artículo. 
meses que acudió a consulta por llanto y dificultad respiratoria, y que fue diagnosticada de neumomediastino asociado a broncoespasmo.

\section{Caso clínico}

Lactante mujer de 12 meses de edad que acudía a consulta por llanto persistente, tos escasa y dificultad respiratoria de unas 10 horas de evolución. Asociaba dos vómitos, rechazo parcial de la alimentación y rinorrea. Entre sus antecedentes personales destacaban tres episodios previos de broncoespasmo. Como antecedente familiar a destacar, la madre padecía asma.
En la exploración clínica se apreciaba: peso de 8,1 kg (P3), talla de $72 \mathrm{~cm}$ (P25), temperatura de $37,4{ }^{\circ} \mathrm{C}$, presión arterial de 101/85 mm Hg, frecuencia cardíaca de 148 latidos por minuto, frecuencia respiratoria de 68 respiraciones por minuto y saturación de oxígeno de $90 \%$. Tenía un buen aspecto general, estaba irritable y no se calmaba en brazos de la madre. Presentaba una respiración entrecortada, con tiraje subcostal e intercostal. En la auscultación respiratoria se objetivaba una buena entrada de aire con sibilancias espiratorias bilaterales y espiración alargada. La auscultación cardíaca era normal. La orofaringe estaba hiperémica,

Figura 1. Signo del diafragma continuo, pequeña cantidad de aire entre mediastino y pleura parietal izquierda, y mínimo enfisema subcutáneo en cuello.

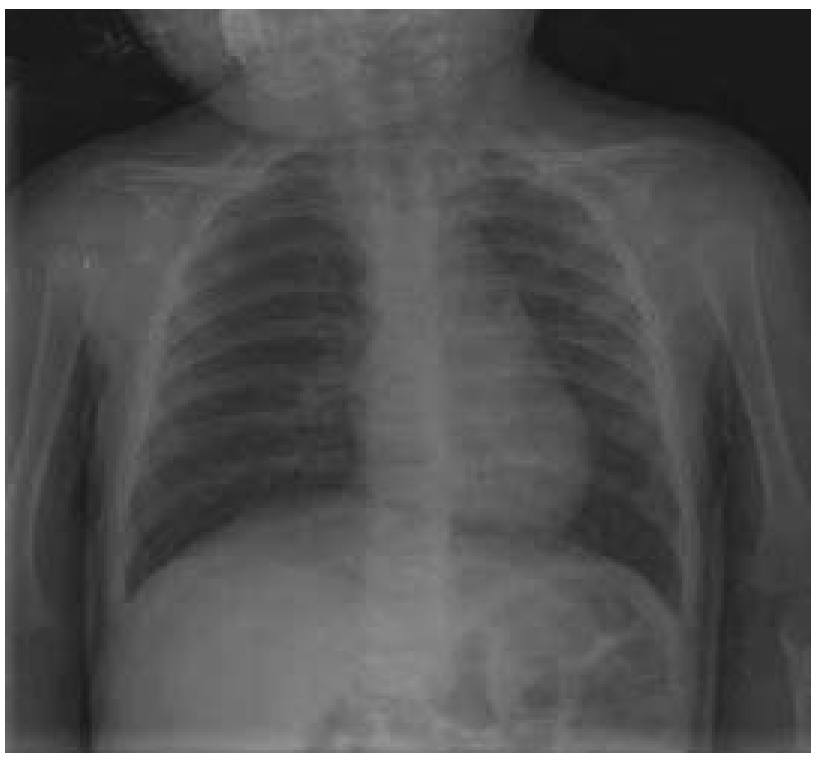


Figura 2. Presencia de aire precardíaco.

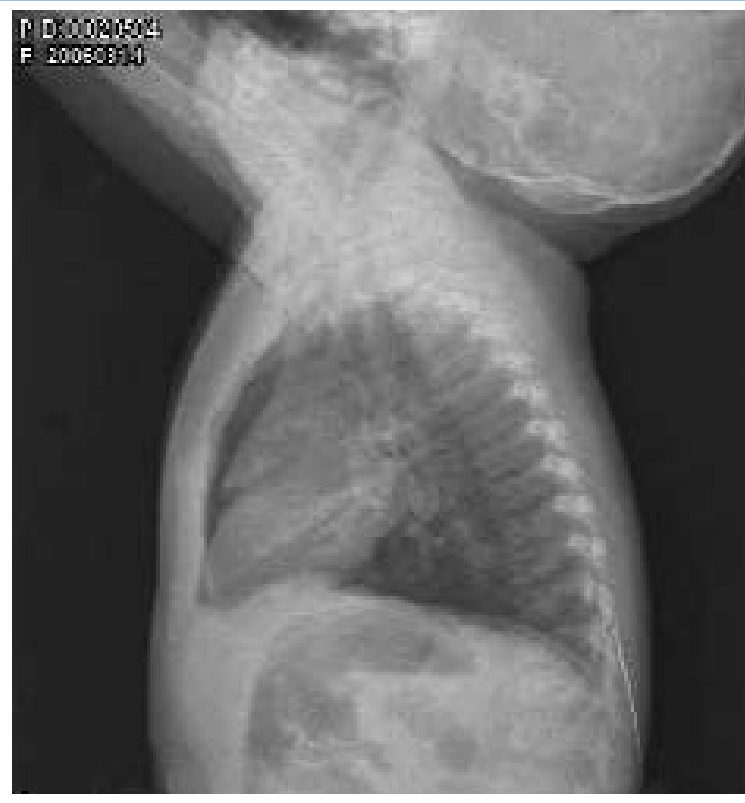

con abundante moco en cavum. En la otoscopia se observaba una hiperemia timpánica bilateral con reflejo luminoso conservado. El resto de la exploración era normal.

Se le realizaron radiografías de tórax anteroposterior y lateral (figuras 1 y 2) en las que se objetivó atrapamiento aéreo con presencia de aire anterocardíaco, signo del diafragma continuo y un mínimo enfisema subcutáneo en cuello. Se llegó con ello al diagnóstico de neumomediastino asociado a broncoespasmo y se ingresó en el hospital. Se le administró tratamiento con oxígeno mediante gafas nasales según necesidades, salbutamol inhalado y prednisolona por vía oral. La evolución clínica fue buena, al tercer día no había signos ni síntomas de dificultad respiratoria, y en la radiografía de tórax de control se apreciaba una disminución del tamaño del aire en mediastino, por lo que fue dada de alta. La prueba de cloruros en sudor fue normal.

\section{Discusión}

El neumomediastino se define como la presencia de aire en el mediastino. Se puede clasificar como neumomediastino médico, traumático e iatrogénico (secundario a intervención quirúrgica, técnicas diagnósticas o terapéuticas). Dentro del 
neumomediastino médico se incluye el espontáneo y el que se presenta asociado a una enfermedad de base predisponente.

El neumomediastino médico es más frecuente en adultos jóvenes y, sobre todo, en varones ${ }^{2-4}$. En niños su hallazgo es extremadamente raro fuera del período neonatal. En el ámbito hospitalario supone el $0,06 \%$ de los ingresos en un servicio de Pediatría ${ }^{5}$.

Las causas más frecuentes del neumomediastino médico en niños son las exacerbaciones del asma (59-75\%), seguido de las infecciones (16-28\%), como las infecciones de vías aéreas superiores, bronquiolitis, neumonía intersticial bilateral, etc. ${ }^{5,6}$. Otras posibles causas son la presencia de un cuerpo extraño en la vía respiratoria o esófago, la fibrosis quística, la inhalación de drogas, el ejercicio físico (sobre todo natación, tenis, culturismo, etc.), la deglución forzada, etc. ${ }^{2,4,6,7}$. Por tanto, aquellas enfermedades o situaciones que predisponen a la realización de maniobras de Valsalva por medio de tos vigorosa, vómitos o esfuerzos respiratorios intensos son las que con más probabilidad pueden causar un neumomediastino.

En la patogenia del neumomediastino es importante la obstrucción respiratoria transitoria aguda, que lleva a que los al- véolos terminales estén sobredistendidos y se puedan romper con facilidad por el aumento de presión y/o tener la pared debilitada por la enfermedad de base. El aire sale a través de las paredes alveolares lesionadas y entra en los espacios intersticiales perivasculares y peribronquiales, dando lugar a un enfisema pulmonar intersticial. El aire progresa hacia el hilio y sale al mediastino. Gran parte de este aire asciende al cuello por los espacios carotídeo, retrofaríngeo o prevertebral, o bien a lo largo de la tráquea y planos musculares. Otra parte del aire se extiende por el tejido graso subcutáneo, dando lugar al enfisema subcutáneo ${ }^{8}$.

Los síntomas más frecuentes son el dolor torácico y la disnea, aunque también pueden quejarse de dolor de cuello, tos, debilidad y disfagia. Menos frecuentes son el dolor de espalda, hombro y abdomen, así como el cuello hinchado. El hallazgo físico más común y que suele ayudar a llegar al diagnóstico es el enfisema subcutáneo. Con frecuencia más variable según las series se puede dar también el signo de Hamman, que consiste en la auscultación de una crepitación sincrónica con los latidos cardíacos ${ }^{2,4,7}$.

La confirmación diagnóstica del neumomediastino se realiza por medio de la radiografía simple de tórax. Los signos radiográficos son el enfisema subcutá- 
neo, el signo de la vela del timo (el timo se eleva por la presencia de aire), el neumoprecardio (presencia de aire delante del pericardio), el signo del anillo alrededor de la arteria pulmonar o sus ramas (el aire está alrededor de la arteria), el signo de la arteria tubular (aire adyacente a las ramas principales de la aorta), el signo de la doble pared bronquial (aire dentro y fuera del bronquio), el signo del diafragma continuo (aire atrapado detrás del pericardio), el signo extrapleural (aire entre la pleura parietal y el diafragma o el mediastino) y la presencia de aire en el ligamento pulmonar'. El neumomediastino y el neumotórax medial pueden ser en todo semejantes en la radiografía simple realizada en supino; pero la proyección lateral en supino permite diferenciarlas fácilmente, pues el aire pleural puede moverse "libremente", algo que no ocurre con el aire en el mediastino.

La evolución es generalmente benigna y autolimitada, con la reabsorción espontánea del aire mediastínico. Por ello, el tratamiento se limita generalmente a reposo durante 8-10 días y analgésicos para el dolor torácico. Normalmente se mantiene al paciente ingresado en observación, dando el alta en pocos días si no se detecta un aumento del aire en mediastino ni complicaciones ${ }^{3}$. En caso de asociarse a otro proceso, como el asma, se trata con las medidas apropiadas. La complicación más frecuente es el neumotórax, que solo ocasionalmente precisa de tubo de drenaje $e^{6,7}$.

El síntoma principal por el que acudía nuestra paciente era el llanto. Tras llegar al diagnóstico fue interpretado como causado por el dolor torácico. Aunque no sea lo habitual, a los padres no les había llamado la atención de una forma especial la dificultad respiratoria de la niña. Esto puede ser debido a varios factores, uno de ellos es que el llanto es un síntoma mucho más perceptible que otros, como el tiraje o la taquipnea. Otros factores pueden ser el bajo nivel sociocultural y la escasa educación sanitaria. Estos pacientes eran muy frecuentadores de los servicios de urgencias hospitalarios, sobre todo en los broncoespasmos previos de la paciente, pero posteriormente no habían acudido en ningún caso a la consulta del centro de salud para su control. Por tanto, no habían recibido ningún tipo de educación respecto al asma del lactante que presentaba la niña y, por tanto, no conocían los signos y síntomas de presentación del asma y cómo actuar en cada caso. Quizá un adecuado tratamiento de la crisis hubiera evitado el posterior desarrollo del neumomediastino.

La frecuencia del neumomediastino en pacientes con una reagudización del as- 
ma es de 0,2 a 0,3\% de los $\operatorname{casos}^{6}$. Dada la rareza del neumomediastino y que, por tanto, pocas veces pensamos en él, queremos enfatizar que ante un lactante o niño pequeño con un broncoespasmo y llanto persistente hay que pensar en esta entidad y realizar una radiografía de tórax para descartarla.

\section{Bibliografía}

1. Vitoria Miñana I. Llanto del lactante. En: Bras i Marquillas J, De la Flor i Brú JE, Torregrosa Bertet MJ, Van Esso Arbolave DL, editores. Pediatría en Atención Primaria 2. ${ }^{a}$ ed. Barcelona: Masson; 2005. p. 437-42.

2. Miura $H$, Taira $O$, Hiraguri $S$, Ohtani $K$, Kato $\mathrm{H}$. Clinical features of medical pneumomediastinum. Ann Thorac Cardiovasc Surg. 2003;9: 18891.

3. Panacek EA, Singer AJ, Sherman BW, Prescott A, Rutherford WF. Spontaneous pneumomediastinum: clinical and natural history. Ann Emerg Med. 1992;21:1222-7.

4. Campillo-Soto A, Coll-Salinas A, Soria-Aledo $\checkmark$. Neumomediastino espontáneo: estudio des- criptivo de nuestra experiencia basada en $36 \mathrm{ca}$ sos. Arch Bronconeumol. 2005;41:528-31.

5. Ballesteros S, García Norniella B, Gracia A, Lagunilla $L$, Fernández Menéndez JM, Matesanz JL. Neumomediastino no traumático en la edad pediátrica. Bol Pediatr. 1998;38:121-4.

6. Damore DT, Dayan PS. Medical causes of pneumomediastinum in children. Clin Pediatr. 2001;40:87-91.

7. Abolnik I, Losson IS, Breuer R. Spontaneous pneumomediastinum. A report of 25 cases. Chest. 1991;100:93-5.

8. Furuse M. Pneumomediastinum: what can we learn? Intern Med. 1998:37:802-3.

9. Zylak CM, Standen JR, Barnes GR, Zylak CJ. Pneumomediastinum revisited. Radiographics. 2000;20:1043-57. 